\title{
Solvent Extraction of Superfine Pulverized Coal. Part 1. Composition of the Extract
}

\author{
Jiaxun Liu ${ }^{\dagger}$, Yuanzhen Jiang ${ }^{\dagger}$, Xue Jiang $*,+$ Hai Zhang ${ }^{\dagger}$, Xiumin Jiang*, \\ ${ }^{\dagger}$ School of Mechanical Engineering, Shanghai Jiao Tong University, Shanghai 200240, China \\ *School of Life Sciences, East China Normal University, Shanghai 200241, China
}

Pages: 4, Figures: 3, Tables: 1 .

Contents:

Fig.S1. Particle size distributions of raw coal samples

Fig.S2 The representative chromatograms of raw coal samples based on SPME-GC-MS

Fig.S3 The representative chromatograms of coal extract based on GC-MS

Table S1. Ash content (ar, wt\%) in raw coal and residue samples

*Corresponding author. Tel: +86 213420 5681; +86 2154344867.

E-mail address: xiuminjiang@sjtu.edu.cn (X.M. Jiang);

xjiang@,bio.ecnu.edu.cn (X. Jiang).

Notes: The authors declare no competing financial interest. 


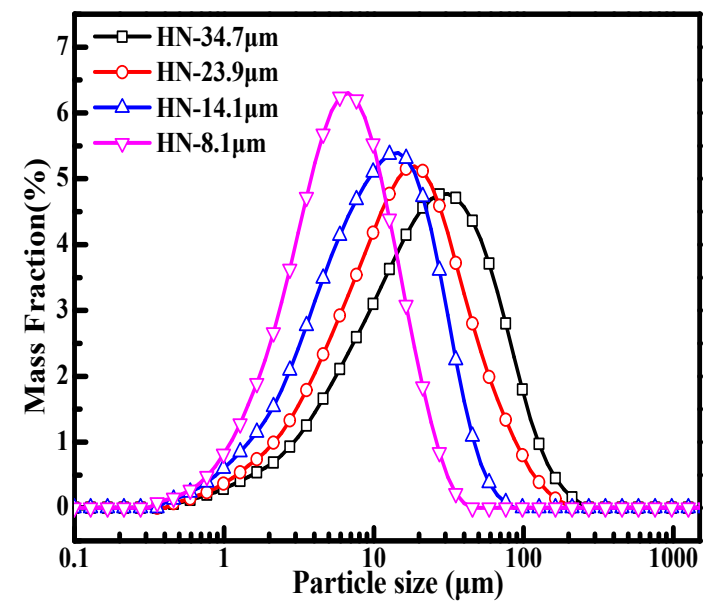

a) HN coal

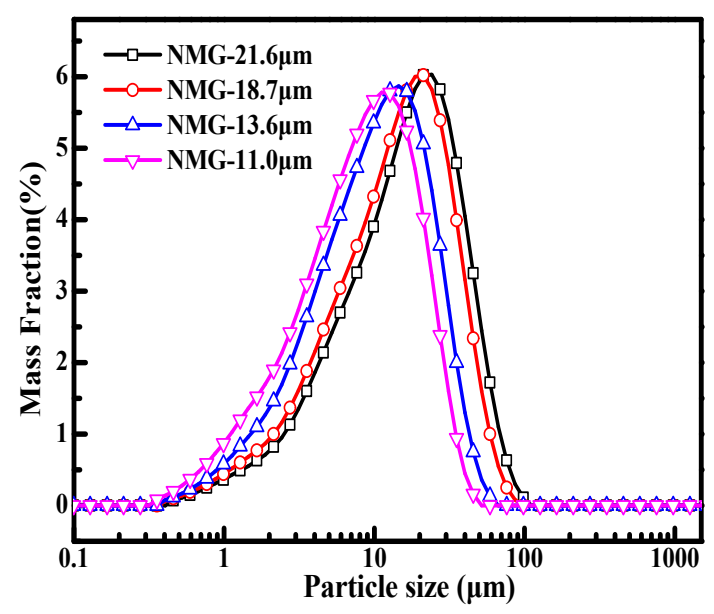

b) NMG coal

Fig. S1. Particle size distributions of raw coal samples 

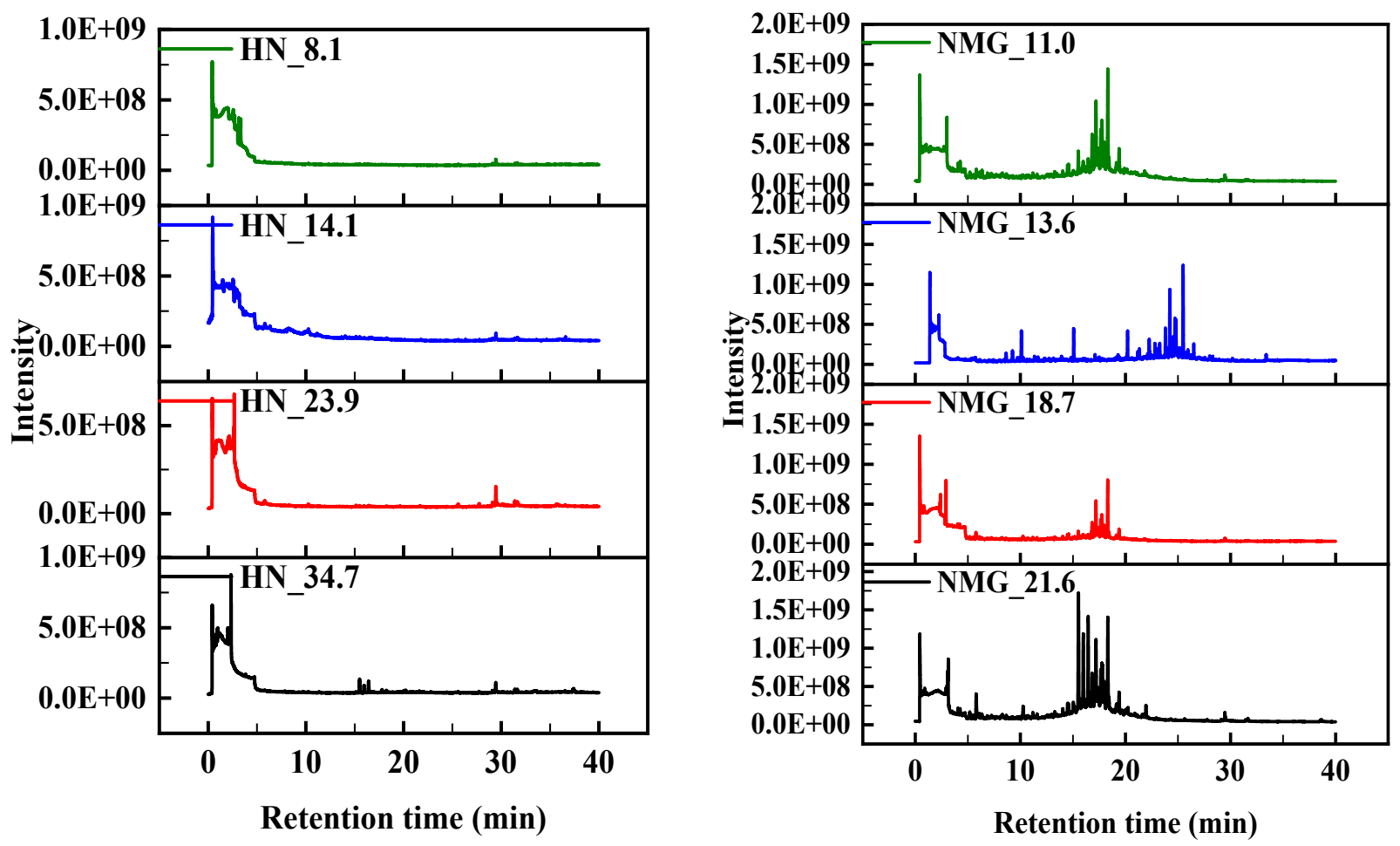

Fig.S2 The representative chromatograms of raw coal samples based on SPME-GC-MS 


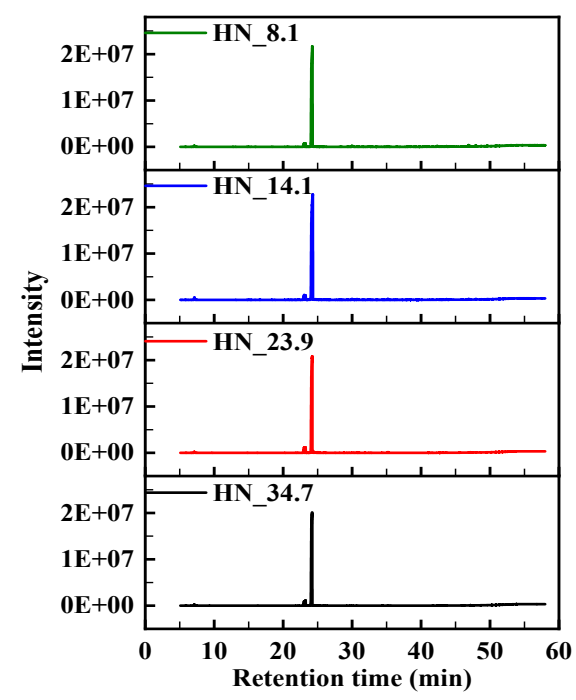

a) $\mathrm{HN}-\mathrm{THF}$

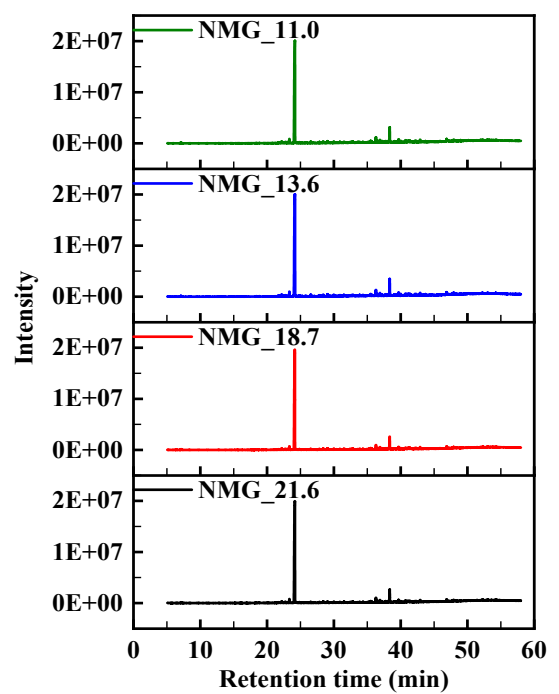

c) NMG-THF

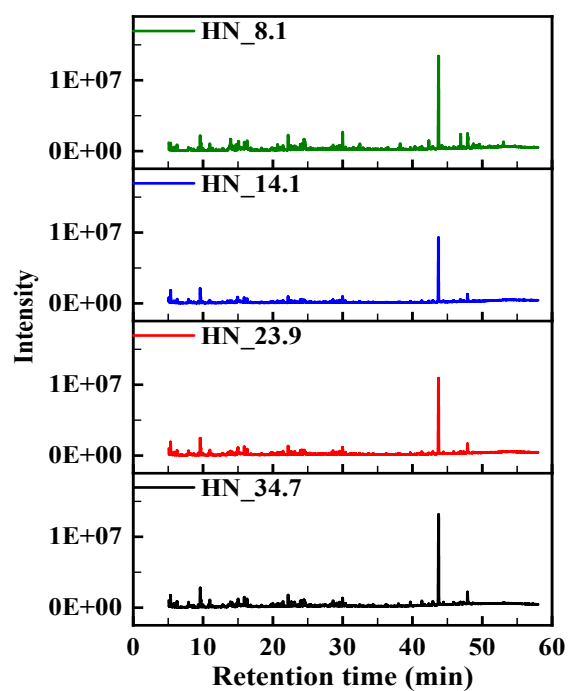

b) HN-PY

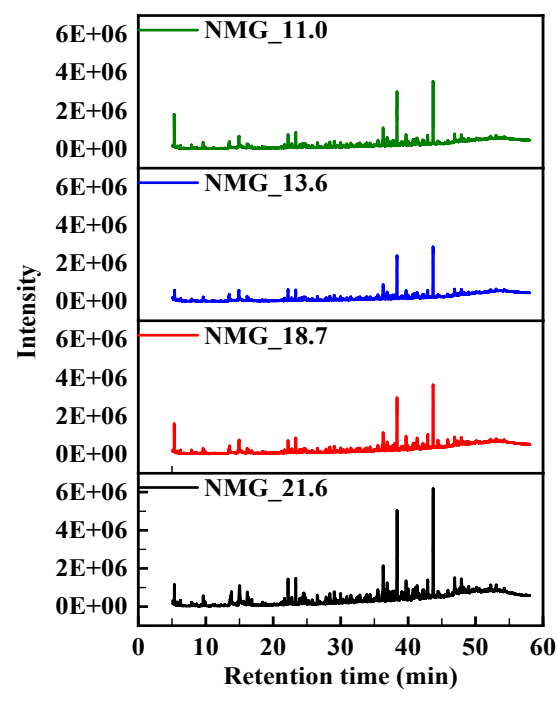

d) NMG-PY

Fig.S3 The representative chromatograms of coal extract based on GC-MS 
Table S1. Ash content (ar, wt\%) in raw coal and residue samples

\begin{tabular}{cccc}
\hline Sample & Raw coal & THF residue & PY residue \\
\hline HN_34.7 & 12.51 & 12.43 & 12.54 \\
HN_23.9 & 12.60 & 12.55 & 12.63 \\
HN_14.1 & 12.89 & 12.83 & 12.85 \\
HN_8.1 & 13.50 & 13.26 & 13.37 \\
NMG_21.6 & 5.32 & 5.34 & 5.35 \\
NMG_18.7 & 5.84 & 5.85 & 5.84 \\
NMG_13.6 & 5.06 & 5.07 & 5.08 \\
NMG_11.0 & 6.25 & 6.35 & 6.35 \\
\hline
\end{tabular}

